\title{
Calculation Model of Preposition Distance of Electronic Toll Collection Signs Based on Lane Changing Behavior
}

\author{
Hao Zhang ${ }^{1,2}$, Changjian Zhang ${ }^{1}$, Ying Zhang ${ }^{1}$, Jinhang Ma ${ }^{1}$, Jie He ${ }^{1, a}$, Ziyang Liu ${ }^{1}$, Xintong Yan ${ }^{1}$ and Qiong Hong ${ }^{3}$ \\ ${ }^{1}$ School of transportation, Southeast University, 211189 No. 2, Southeast University Road, Nanjing, China \\ ${ }^{2}$ School of traffic engineering, Huaiyin institute of technology, 223003 No.1, Meichengdonglu, Huaian, China \\ ${ }^{3}$ Business School, Jiangsu Vocational College of Electrics and Information, 223003 No.3, Meichengdonglu, Huaian, China
}

\begin{abstract}
ETC and MTC lanes of China's hybrid toll stations have various setting modes. When the vehicles passed through the toll stations, they would face a more complicated lane change process. If the ETC sign was set in the appropriate position in advance, the traffic safety in front of the toll stations would be effectively improved. The paper analyzed the process of lane change in 18 scenes by taking one-way three-lane highway at the upstream of toll station with six lanes as an example. Based on the definition of driver's reaction distance, reading distance and safe distance of action, the theoretical calculation model of ETC sign preposition distance was established. It revealed the functional relationship between ETC lane layout schemes and sign preposition distance, and explored the reasonable setting position of the ETC sign in the full scenes of the lane layout. Finally, a case study of Nanjing toll station on Shanghai-Nanjing Expressway was carried out.
\end{abstract}

\section{Introduction}

The coexistence of ETC and MTC in the mixed toll station makes the two traffic flows with different characteristics inevitably interfere with each other. Unreasonable location of ETC lane indication signs will also cause frequent lane change, emergency lane changes and other dangerous situations. The reasonable location of ETC lane indicator will give the driver sufficient reaction and operation time, and avoid the phenomenon of emergency lane change, which is of great significance to strengthen the traffic safety construction of the main line toll station.

Scholars in various countries have carried out different levels and depth research in the design of ETC signs in toll stations, mainly focusing on the form, quantity and content of indicator signs, and a small number of studies involving location setting, but they have ignored the safety and particularity of coexistence of ETC and MTC. Albert E. ${ }^{[1]}$ (1997) has made an in-depth study on the setting scheme of toll lane and the standard of mark line; $\mathrm{Pu}$ et.al ${ }^{[2]}$ (2012) establish the optimization model of variable speed limit control, calculated and verified the number and setting distance of variable information boards; Chi et al. ${ }^{[3]}$ (2015) put forward the optimized design scheme of the indicator sign of the unattended toll station of the expressway based on the simulation driving test; Valdes et al. ${ }^{[4]}$ (2016) find that hanging toll station signs over the cockpit simulator has a better guiding effect than roadside signs; Hu et al. ${ }^{[5]}$ (2018) study the

\footnotetext{
${ }^{\mathrm{a}}$ Corresponding author: hejie@seu.edu.cn
}

occlusion probability of roadside signs with the help of VISSIM simulation, and the research results can be used to set the deflection angle and position of roadside traffic signs.

There is no exploration and research on the location setting of ETC signs from the perspective of safety, but it is of great significance to carry out the research on the front distance of ETC signs in the main line toll station considering the lane change.

\section{Analysis of vehicle lane change process in front of ETC toll station}

When the driver sees the indication sign of ETC lane, they would select the corresponding toll lane according to the location of ETC lane and their own needs, they implement lane change operation and complete the payment. This paper takes one-way three lanes and six toll lanes of the basic section of expressway as an example.

The initial location of vehicles in the basic section of expressway and the location of the target payment lane determine the different lane changing process. This paper takes the conservative driver as an example. Table 1 shows 18 specific lane change scenarios. Figure 1 is the schematic diagram of several typical lane change processes, in which the lane numbers of the basic section are $\mathrm{A}, \mathrm{B}$ and $\mathrm{C}$ from the inside to the outside; the lane numbers of the toll lane are 1-6 from the inside to the 
outside; the lane change mode " $a+b$ " represents the vehicle change lanes $a$ times in the basic section and $b$ times in the transition section.

Table 1 Analysis of lane changing process in different scenarios

\begin{tabular}{|c|c|c|c|c|c|c|}
\hline \multirow[t]{2}{*}{ Number } & \multirow[t]{2}{*}{ Plan } & \multicolumn{2}{|c|}{$\begin{array}{c}\text { Basic } \\
\text { expressway } \\
\text { sections }\end{array}$} & \multicolumn{2}{|c|}{$\begin{array}{c}\text { Transition } \\
\text { section of Toll } \\
\text { Plaza }\end{array}$} & \multirow{2}{*}{$\begin{array}{c}\text { Lane } \\
\text { change } \\
\text { mode }\end{array}$} \\
\hline & & $\begin{array}{c}\text { Initial } \\
\text { Lane }\end{array}$ & $\begin{array}{l}\text { End } \\
\text { Lane }\end{array}$ & $\begin{array}{c}\text { Initial } \\
\text { Lane }\end{array}$ & $\begin{array}{l}\text { End } \\
\text { Lane }\end{array}$ & \\
\hline 01 & A-1 & A & A & 1 & 1 & $0+0$ \\
\hline 02 & A-2 & A & B & 2 & 2 & $1+0$ \\
\hline 03 & A-3 & A & B & 2 & 3 & $1+1$ \\
\hline 04 & A-4 & A & $\mathrm{C}$ & 3 & 4 & $2+1$ \\
\hline 05 & A-5 & A & $\mathrm{C}$ & 3 & 5 & $2+2$ \\
\hline 06 & A-6 & A & $\mathrm{C}$ & 3 & 6 & $2+3$ \\
\hline 07 & B-1 & B & A & 1 & 1 & $1+0$ \\
\hline 08 & B-2 & B & B & 2 & 2 & $0+0$ \\
\hline 09 & B-3 & B & B & 2 & 3 & $0+1$ \\
\hline 10 & B-4 & B & $\mathrm{C}$ & 3 & 4 & $1+1$ \\
\hline 11 & B-5 & B & $\mathrm{C}$ & 3 & 5 & $1+2$ \\
\hline 12 & B-6 & B & $\mathrm{C}$ & 3 & 6 & $1+3$ \\
\hline 13 & $\mathrm{C}-1$ & $\mathrm{C}$ & A & 1 & 1 & $2+0$ \\
\hline 14 & C-2 & $\mathrm{C}$ & B & 2 & 2 & $1+0$ \\
\hline 15 & C-3 & $\mathrm{C}$ & $\mathrm{C}$ & 3 & 3 & $0+0$ \\
\hline 16 & C-4 & $\mathrm{C}$ & $\mathrm{C}$ & 3 & 4 & $0+1$ \\
\hline 17 & C-5 & $\mathrm{C}$ & $\mathrm{C}$ & 3 & 5 & $0+2$ \\
\hline 18 & C-6 & $\mathrm{C}$ & $\mathrm{C}$ & 3 & 6 & $0+3$ \\
\hline
\end{tabular}

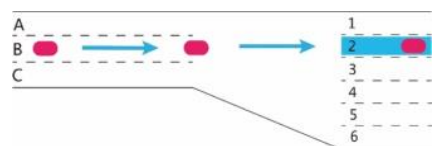

(a) Scheme B-2 lane change process

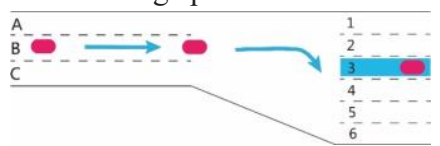

(b) Scheme B-3 lane change process

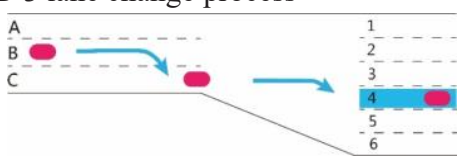

(c) Scheme B-4 lane change process

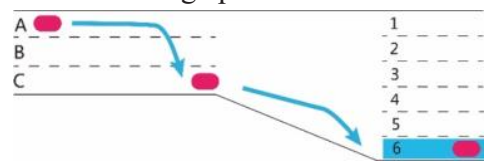

(d) Scheme A-6 lane change process

Figure 1 Several typical lane changing process diagrams

From the above analysis, it can be seen that with the location of ETC lane to the outside, the more lanes ETC users need to cross in the transition section, and in case of scheme A-6, ETC users need to cross the most lanes. When the location of ETC lane is arranged on the inside, ETC users can complete the process of lane changing in the basic section of expressway.

\section{Calculation model of preposition safety distance of Electronic Toll Collection signs}

\subsection{Definition of preposition distance of signs}

According to the above analysis of lane change process, ETC users need to have enough distance to complete a series of lane change operations before passing through the main line toll station.

As shown in Figure 2, it is defined that the distance $L_{l}$ from the start reading point to the action point is the reaction distance, the distance $L_{2}$ from the start reading point to the sign point is the reading distance, the distance L3 from the action point to the completion point is the action safety distance, the distance $L 4$ from the sign point to the completion point is the front distance of the mark, The distance from the starting point to the completion point is the safe distance $L=L_{1}+L_{3}=L_{2}+L_{4}$.

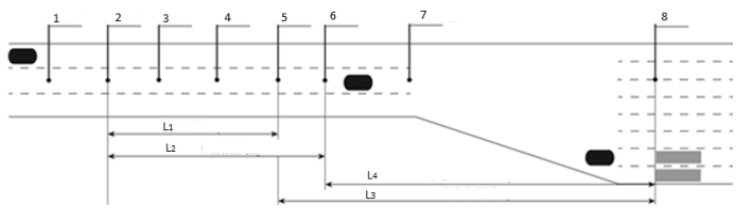

Figure 2 Analysis of vehicle passing through ETC toll station

Points 1-8 represent the visual recognition point, start reading point, reading point, vanishing point, action point, flag point, starting point and completion point of the gradient section respectively.

\subsection{Reaction distance and ETC sign scan distance}

The driver usually have $1.5 \mathrm{~s}$ for sensory time and $1.0 \mathrm{~s}$ for judgment time ${ }^{[6]}$. The reaction distance is calculated according to the following formula:

$$
L_{1}=\frac{V_{1} t_{1}}{3.6}=0.694 V_{1}
$$

$t_{l}$ is the total reaction time, it is noted as $2.5 \mathrm{~s} ; \mathrm{v}_{1}$ is the driving speed of the car. $(\mathrm{km} / \mathrm{h})$

When the human eye distinguishes the text, it needs at least $10-15^{\prime}$ visual angle ${ }^{[7]}$, then the visual recognition distance can be calculated according to the formula of visual distance ${ }^{[8]}$ :

$$
L_{2}=\frac{H}{\alpha} 57.3
$$

$H$ is the height of text in $\operatorname{sign}(\mathrm{cm}) ; \alpha$ is the angle of view. The angle of view here is 1.0 according to the driver's vision requirements, 10 'for $\alpha$. Font height is taken as $50 \mathrm{~cm}$. Then the visual distance is calculated as follows:

$$
L_{2}=\frac{50}{10} \times 57.3=286.5 \mathrm{~m}
$$

\subsection{Driving safety distance}

According to the road characteristics, the driving safety distance is divided into three parts: the basic section of expressway, the gradual change section of toll plaza and the straight line section of toll gate. 


\subsubsection{Calculation of driving distance of basic section of expressway}

There are four stages for the driver to operate the vehicle in the basic section of the expressway: waiting for the insertable gap, making judgment on the insertable gap, adjusting the speed, and moving the vehicle across the lane ${ }^{[9]}$. Set the longitudinal distance of the above four processes as $l_{1}, l_{2}, l_{3}$ and $l_{4}$ respectively.

(1) The distance of the vehicle travels while waiting for the insertable gap: $l_{l}$

In the free flow state, the headway obeys the exponential distribution. In general, the negative exponential function of displacement can be used to solve the waiting time of vehicle to insert the gap ${ }^{[10]}$ : $t_{w}=\overline{H n}=\frac{e^{\lambda(t(t-\tau)}-\lambda(t-\tau)-1}{\lambda \cdot e^{\lambda(t-\tau)}} \cdot \frac{1}{1-e^{-\lambda(t(-\tau)}} \cdot \frac{1-e^{-\lambda(t(-\tau)}}{e^{-\lambda(t(\tau))}}=\frac{1}{\lambda}\left[e^{\lambda(t(t-\tau)}-\lambda(t-\tau)-1\right]$

In the formula, $t$ is critical clearance, generally $4.0 \mathrm{~s} ; \lambda$ is average arrival rate; $\tau$ is the minimum headway, generally $1.2 \mathrm{~s}$.

Set $Q$ here as the traffic flow of expressway, so $\lambda=Q / 3600$. $t_{w}$ for the vehicle to be inserted into the gap. Then the distance of the vehicle waiting to be inserted into the gap can be calculated:

$$
l_{1}=\frac{V_{1}}{3.6} \cdot t_{w}=0.278 V_{1} \cdot t_{w}
$$

(1) Judge the distance of the vehicle within the time for the insertable gap: $l_{2}$

The distance that the vehicle passes in the process of judgment is distance that can be inserted. In this process, the vehicle has been driving at the speed $V_{h}$, and the time required in the process of adjusting the speed is $t_{2}$ :

$$
t_{2}=\frac{V_{h} t_{w}}{\Delta v}
$$

$V_{h}$ is the speed when judging the insertable gap; $\Delta v=V_{1}-V_{h}$ is relative velocity, commonly $V_{h l}=0.76 V_{1}^{[11]}$.

$$
l_{2}=V_{1} \cdot t_{2}=\frac{V_{1}}{3.6} \cdot \frac{V_{h} t_{w}}{\Delta v}=\frac{V_{1}}{3.6} \cdot \frac{0.76 V_{1} t_{w}}{V_{1}-0.76 V_{1}}=0.879 V_{1} t_{w}(7)
$$

(2) Distance that the driver adjusts the speed of the vehicle and waits during the lane change: $l_{3}$

After judging that the gap can be inserted, the driver adjusts the speed and the body angle for the lateral lane change, which generally takes $1.5 \mathrm{~s}$, but considering the influence of the road traffic environment and the driver's own quality condition on the time, the response time is determined as $2.5 \mathrm{~s}^{[12]}$, so the distance is:

$$
l_{3}=\frac{V_{1} t_{3}}{3.6}=0.694 V_{1}
$$

$t_{3}$ is the time to adjust the vehicle speed.

(3) The distance of vehicle moving across the lane: 14

After the driver is ready to change lanes, they begin the process of changing lanes. Assuming that one lane is traversed at a time, the time required for the vehicle to traverse is:

$$
t_{4}=\frac{d}{J}
$$

$d$ is the lane width of one basic section; $J$ is the average traverse speed, generally $1 \mathrm{~m} / \mathrm{s}$. If the running speed of the vehicle in the process of lane change is $V_{l}(\mathrm{~km} / \mathrm{h})$, the longitudinal running distance of the vehicle when it moves horizontally is:

$$
l_{4}=\frac{V_{1}}{3.6} \cdot t_{4}=\frac{V_{1}}{3.6} \cdot \frac{d}{J}=0.278 V_{1} d
$$

Assuming that $n$ is the number of continuous lane changes in the basic vehicle section, the calculation model of the action distance of the basic expressway section is as follows:

$$
L^{\prime}=N\left(l_{1}+l_{2}+l_{3}+l_{4}\right)
$$

\subsubsection{Moving distance of vehicles in transition section}

Different from the basic section of expressway, if the lane is changed $n$ times in the gradual change section of toll plaza, the course of action includes: one time waiting for inserting clearance, one time judging inserting clearance, one time adjusting speed and $n$ times vehicle moving crosswise for lane changing, in addition, there are also differences in vehicle speed. The gradient rate of the transition section in the toll plaza is $\varphi=H / L$, the vehicle speed is $V_{2}$, and the track rotation angle is $\theta$, as shown in Figure 3.

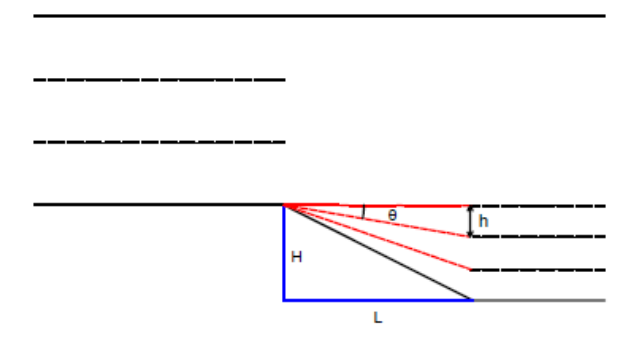

Figure 3 Schematic diagram of trajectory angle

Suppose that there are 3 more lanes in the transition section than in the basic section, and the vehicle crosses 1 Lane in the transition section, So:

$$
\tan \theta=\frac{h}{L}=\frac{3 h}{3 L}=\frac{H}{3 L}=\frac{\varphi}{3}
$$

$h$ is the width of a single lane in the transition section.

When the transition section has m more lanes than the basic section, and the vehicle crosses $n$ lanes in the transition section:

$$
\tan \theta=\frac{n \varphi}{m}
$$

And it can be transformed:

$$
\theta=\arctan \frac{n \varphi}{m}
$$

Therefore, the calculation model of vehicle moving distance in the gradual change section of toll plaza is as follows: 


$$
l_{5}=0.278 V_{2} \cdot t_{w}{ }^{\prime}+0.879 V_{2} t_{w}{ }^{2}+0.694 V_{2}+0.278 V_{2} n d \cos \theta
$$

$t_{w}$ is the time for vehicles in the transition section to wait for the gap to be inserted.

\subsubsection{Action distance of straight-line section of toll gate: $I_{6}$}

In order to ensure the reliability of identification, the general traffic speed of ETC lane is $20 \mathrm{~km} / \mathrm{h}$. Considering the high speed of ETC users, it is generally stipulated that the action distance $l_{6}$ of the straight-line section of the toll gate shall not be less than $35 \mathrm{~m}$.

\subsubsection{Calculation of the safe distance from the starting point to the completion point}

Therefore, the safe distance calculation model for ETC lane of main toll station is as follows:

$$
\begin{aligned}
& L=L_{2}+L_{4}=L_{1}+L_{3}=L_{1}+N\left(l_{1}+l_{2}+l_{3}+l_{4}\right)+l_{5}+l_{6} \\
& =0.694 V_{1}+N\left(0.278 V_{1} t_{w}+0.879 V_{1} t_{w}+0.694 V_{1}+0.278 V_{1} d\right) \\
& +0.278 V_{2} \cdot t_{w}{ }^{\prime}+0.879 V_{2} t_{w}{ }^{\prime}+0.694 V_{2}+0.278 V_{2} n d \cos \theta+l_{6}
\end{aligned}
$$

\subsection{Calculation of preposition distance of electronic toll collection signs}

The calculation model of the preposition safety distance of ETC sign is as follows:

$$
\begin{aligned}
L_{4}=L-L_{2}= & 0.694 V_{1}+N\left(0.278 V_{1} t_{w}+0.879 V_{1} t_{w}+0.694 V_{1}+0.278 V_{1} d\right) \\
& +0.278 V_{2} \cdot t_{w}{ }^{\prime}+0.879 V_{2} t_{w}{ }^{\prime}+0.694 V_{2}+0.278 V_{2} n d \cos \theta+l_{6}-L_{2} \\
= & 0.694 V_{1}+N\left(1.157 V_{1} t_{w}+0.694 V_{1}+0.278 V_{1} d\right) \\
& +\left[1.157 V_{2} t_{w}{ }^{\prime}+0.694 V_{2}+0.278 V_{2} n d \cos \left(\arctan \frac{n \varphi}{m}\right)\right]+l_{6}-L_{2}
\end{aligned}
$$

According to Announcement No. 35 of 2007 on technical requirements for toll road network toll collection, the range of transition rate $\varphi$ is $1 / 5 \sim 1 / 7$.

Generally, the more complex the lane change process is, the greater the lane change distance is required. The paper only discusses the situation of lane change of vehicles in the basic road section. When $N \neq 0, n=0$, the basic road section will be changed normally, and the transition section will not be changed. it is only necessary to make a uniform deceleration movement, the average deceleration is $a\left(\mathrm{~m} / \mathrm{s}^{2}\right)$, and the calculation formula according to the kinematic distance is as follows:

$$
l_{5}=\frac{V_{1}{ }^{2}-V_{2}^{2}}{3.6^{2} \times 2 a}
$$

From this, we can get the safety distance of ETC indication signs corresponding to the specific layout scheme of ETC toll lane $(f(N, n) \max )$ :

$$
f(N, n)_{\max } \begin{cases}0.694 V_{1}+N\left(1.157 V_{1} t_{w}+0.694 V_{1}+0.278 V_{1} d\right) & \\ +\left[1.157 V_{2} t_{w}+0.694 V_{2}+0.278 V_{2} n d \cos \left(\arctan \frac{n \varphi}{m}\right)\right]+l_{6}-286.5 & N \neq 0, n \neq 0 \\ 0.694 V_{1}+N\left(1.157 V_{1} t_{w}+0.694 V_{1}+0.278 V_{1} d\right) & \\ +\frac{V_{1}^{2}-V_{2}^{2}}{3.6^{2} \times 2 a}+l_{6}-286.5 & N \neq 0, n=0\end{cases}
$$

$a$ is the average deceleration of the uniform deceleration movement, taking $2.5 \mathrm{~m} / \mathrm{s}^{2}$. The number of lane changes in the basic section of each lane $N$ and the number of lanes crossed by the transition section of the toll plaza $n$ are calculated according to the following formula:

$$
\left\{\begin{array}{cc}
n=0, N=\max (M-i, i-1) & i \leq M \\
n=i-M, N=M-1 & i>M
\end{array}\right.
$$

$M$ is the number of one-way lanes in the basic expressway section, $I$ is the total number of one-way toll lanes, $i$ is the location number of toll lanes, from the inside to the outside, $1,2,3 \ldots I$.

\section{Case study}

\subsection{Analysis of lane change of Nanjing toll station on Shanghai-Nanjing Expressway}

In this paper, we analyze the Nanjing toll station of Shanghai-Nanjing Expressway as an example. The basic section of the main line toll station expressway is oneway 4-lane. The toll lanes from the inside to the outside are 3 manual toll lanes, 2 ETC toll lanes, 7 manual toll lanes, and 2 green passages, as shown in Figure 4. It is required that the speed to pass the ETC lane is $20 \mathrm{~km} / \mathrm{h}$, the speed is $40 \mathrm{~km} / \mathrm{h}$ in the transition section, and the speed in the basic section of the highway is $60 \mathrm{~km} / \mathrm{h}$.

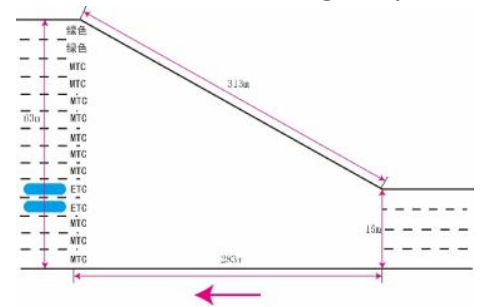

Figure 4 Schematic diagram of the toll station

Mark the four lanes of the basic section of the highway from the inside to the outside as lane 1, 2, 3, 4. Mark the 2 ETC lanes from inside to outside as lane a and $b$. Therefore, the most complicated lane change process when ETC users pass through the toll station is that change from lane 1 to lane $b(3+1$ lane change mode $)$ as shown in Figure 5. We would calculate for this program in the following chapters. 


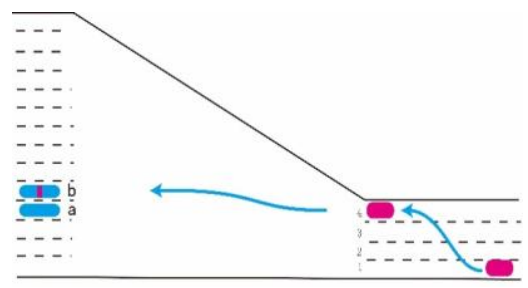

Figure5 Lane change process under the most complicated ETC lane setting scheme

\subsection{Calculation of preposition distance of Electronic Toll Collection Signs}

According to the investigation of Nanjing toll station of Shanghai-Nanjing Expressway, $V_{1}$ is $60 \mathrm{~km} / \mathrm{h}, V_{2}$ is $40 \mathrm{~km} / \mathrm{h}$. According to formula (4), in the basic section of expressway $t_{w}=1.98 \mathrm{~s}$, in the toll plaza gradient $t^{\prime}{ }_{w}=1.5 \mathrm{~s}$. The width of the lane $\mathrm{d}=3.75 \mathrm{~m}$, and the gradient rate

$$
\varphi=\frac{48}{283}=0.17, l_{6}=55 \mathrm{~m} .
$$

When the driver continuously changes lanes on the basic section of the expressway, $N=3, n=1, m=10$. At this time, the leading distance of the ETC sign:

$L_{4}=0.694 V_{1}+N\left(1.157 V_{1} t_{w}+0.694 V_{1}+0.278 V_{1} d\right)+$

$$
\begin{aligned}
& \left(1.157 V_{2} t_{w}{ }^{\prime}+0.694 V_{2}+0.278 V_{2} n d \cos \left(\arctan \frac{n \varphi}{10}\right)\right)+l_{6}-286.5 \\
= & 0.694 \times 60+3 \times(1.157 \times 60 \times 1.98+0.694 \times 60+0.278 \times 60 \times 3.75) \\
& +\left(1.157 \times 40 \times 1.5+0.694 \times 40+0.278 \times 40 \times 1 \times 3.75 \times \frac{1}{\sqrt{1+\left(\frac{0.17}{10}\right)^{2}}}\right)+55-286.5 \\
\approx & 674(\mathrm{~m})
\end{aligned}
$$

In theory, the front distance of the toll station ETC indicator should be $\geqslant 674 \mathrm{~m}$.

\subsection{Safety analysis and recommendations}

In actuality, the Preposition distance of the ETC signs is $420 \mathrm{~m}$. That may cause traffic conflicts and safety hazards. Combining the calculation and analysis results, the following suggestions are provided:

(1) Adjust the ETC lane layout plan. Arrange ETC lanes in lane 1 to lane 4 to avoid or reduce ETC users' lane changes in the gradual change section of the toll plaza, thereby reducing potential lane change risks.

(2) The position of the indicator for the ETC lane position is advanced for 675 meters and is set multiple times within a certain distance. That could ensure most vehicles change lanes to the target lane in advance and reduce the temporary emergency lane changes during the gradual transition.

\section{Summary and prospect}

The thesis explains the impact of the ETC lane setting on the vehicle lane changing process in conjunction with the driver 's lane changing process when passing through the toll station. The safety distance is defined based on the process of driver identification ETC signs, lane change deceleration, etc. And it is divided into three parts: reaction distance, sign reading distance, and action safety distance for calculation.

The action safety distance which is divided into three parts: the basic highway section, the gradient section of the toll plaza, and the straight section of the toll gate was analyzed and calculated based on the lane change theory. Finally, the theoretical calculation model of the ETC indicator leading distance was established. And the safety inspection of the Nanjing-Shanghai toll station of the Shanghai-Nanjing Expressway was carried out based on this model. It is found that the preposition distance of the existing ETC is insufficient and there is a potential safety hazard.

\section{Acknowledge}

This research is supported by the National Natural Science Foundation of China (71874067 \& 51778141), "Six Industry Talent Peak Project" fund of Jiangsu Province (RJFW049 \& JNHB115), Green and Blue Project fund of Jiangsu Province, Philosophy and Social Science fund for colleges in Jiangsu Province :2017SJB1641 \& 2018SJA1649, Natural science fund for colleges in Jiangsu Province: 17KJB58001.

\section{References}

1. Albert, Schaufler. Toll plaza design $[\mathrm{M}]$. U.S.A: Transportation Research Board, 1997:8-40.

2. $\mathrm{Pu}$ Yun, $\mathrm{Hu} \mathrm{Lu}$ and Jiang Yangsheng. Variable speed limit control of Expressway main toll station [J]. Journal of Transportation Engineering, 2012, 12(5):119-126.

3. Chi Jinghao, Zhao Xiaohua and Rong Jian. Study on the design scheme of Expressway unattended toll station indication sign based on driving simulator [J]. Traffic information and safety, 2015(6):114-119.

4. Valdés D, Colucci B, Fisher D L, et al. Driving Simulation of the Safety and Operation Performance at a Freeway Toll Plaza[J]. Transportation Research Record: Journal of the Transportation Research Board, 2016 (2602): 129-137.

5. Hu Shaorong, Ma Rongguo and Li Miaomiao. Study on occlusion probability of roadside traffic signs of multi Lane Expressway [J]. China Journal of Highway and Transport, 2018, 31(3):116-122,150.

6. Zhang Boming. A study on the visual recognition of Chinese characters in traffic signs [J]. Highway transportation technology, 1993, 2(10): 40-46.

7. Guo $\mathrm{Fu}$ and Qian Xing. Human factors engineering [M].Beijing: China Machine Press, 2000.

8. Li Zhongyuan and Li Shuangshuang. Research on the design of traffic information display screen based on human factors engineering [J]. Intelligent building and city information, 2010(2):40-43. 
9. Wu Dehua. Analysis and Research on the minimum safe distance between tunnel and interchange [D]. Nanjing: Southeast University, 2014.

10. Li Wenquan, Wang Wei and Zhou Ronggui. Distribution characteristics of headway in Lane 1 of Expressway merging area [J]. Highway transportation technology, 2003, 20(1): 114-117.
11. Cao Hehong. Study on the speed change lane of Expressway Interchange [D]. Beijing: Beijing University of technology, 1999.

12. Ren Futian. Traffic engineering psychology $[\mathrm{M}]$. Beijing: Beijing University of Technology Press, 1999 